\title{
COMPUTATIONAL VALIDATION OF DISSIPATIVE DEVICE FOR THE SEISMIC UPGRADE OF HISTORIC BUILDINGS
}

\author{
Victor Melatti ${ }^{1}$, Dina D'Ayala ${ }^{1}$, Erica Modolo ${ }^{2}$ \\ ${ }^{1}$ University College London Gower St, Bloomsbury, London WC1E 6BT \\ victor.melatti.16@ucl.ac.uk, d.dayala@ucl.ac.uk \\ ${ }^{2}$ University of Padua Via 8 Febbraio 1848, 2, 35122 Padova PD, Italy \\ erica.modolo@studenti.unipd.it
}

\begin{abstract}
The lack of effective connections between sets of perpendicular walls often determines the local or global failure of heritage structures in case of seismic event. In order to address this problem, the paper focuses on retrofitting systems for masonry structures undergoing seismic load. Particularly, it concerns the behavior of two different types of anchoring stabilization systems that avoid the out-of-plane collapse mechanism of a masonry wall and restore the boxlike behavior. The response of the corner connection between two walls was investigated by numerical simulations. Initially, the model was tested in its original configuration to determine the type and the extent of the failure mode when a base acceleration is imposed. Afterwards, a traditional steel anchoring system was implemented in the corner connection to prevent the relative detachment of the walls for the same seismic input. Finally, a friction-based dissipative device was added to the steel ties to provide ductility to the system by allowing for a controlled relative displacement of the walls and reducing the concentration of stresses. The computational analysis showed that implementation of a dissipative device integrated with traditional steel anchors is effective in delaying the complete failure of the anchoring system. Moreover, it provides energy dissipation capacity through controlled displacement, reducing the stress in the surrounding material and allowing the model to withstand a larger intensity input.
\end{abstract}

Keywords: Dissipative devices, Friction, Masonry, Energy Dissipation 


\section{INTRODUCTION}

In the last decades, a variety of systems able to dissipate seismic input energy has been designed, enhancing the global response of structures in earthquake prone areas [1] [2]. Historic buildings represent a singular case: the implementation of systems based on the modern concept of ductility and energy dissipation is often in contrast with the limitations promulgated to protect the aesthetic and structural integrity of architectural assets. Therefore, the common retrofitting practice still relies on traditional techniques of intervention and historic centres are still considerably affected by earthquakes [3] [4].

It is recognised that poor-quality connections between orthogonal structural elements greatly affect the dynamic performance of heritage masonry structures causing the relative detachment of masonry panels and ultimately the out-of-plane failure of the structure [5]. A way of tackling this issue is to insert steel cross-ties at the corner of two perpendicular walls in order to restore the box-like behaviour and distribute the seismic forces through all the resisting elements. This practice is not exempt from drawbacks, as the increased local stiffness at the corners might lead to high-stress concentration in case of seismic events and consequently to severe damage to the valuable parent material in which the cross-ties are embedded [6].

Therefore, in the last decades alternative systems have been developed to provide energy dissipation capacity to heritage structures, in line with the modern principle of performancebased design. This approach optimizes the structure for different seismic action level, encouraging the use of devices able to provide ductility by shifting the response of the structure from the elastic-only range to an elasto-plastic one [7].

These innovative systems are tailored for historic masonry structures as they reflect the conservation principles enshrined in international and national guidelines such as the ICOMOS/ISCARSAH principles [8] and the Italian DPCM 2011 [9]. According to these documents, the benefits of possible upgrade interventions in terms of seismic performance must be weighed against the impact on the original aesthetic and structural authenticity of the building. A variety of systems are available [10],[11], [12], but technical codes are vague on design procedures and their implementation hardly follows.

The present work proposes an innovative dissipative anchoring system to improve the global response of historic structures to seismic events. The proposed friction-based anchoring system offers an innovative alternative to traditional systems for the repair and strengthening of heritage structures, using performance-based design principles, i.e. control of displacements and reduction of accelerations and concentration of stress. The innovative system comprises a friction device, shown in Figure 1a, connected to longitudinal stainless steel bars grouted within the thickness of the walls. The assembly is designed to be inserted at the connection between perpendicular walls (Figure 1b): the stainless steel profiles improve the box-like behaviour of the building, while the device allows for a relative displacements between orthogonal sets of walls and the dissipation of the seismic energy input to the structure. The system aims at preventing typical failure mechanism of reinforced and unreinforced masonry buildings under seismic loading, such as punching failure and excessive crack opening. 
a)

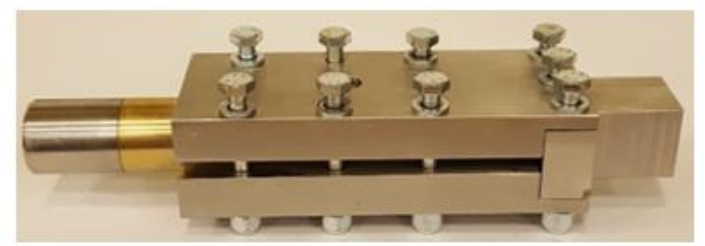

b)

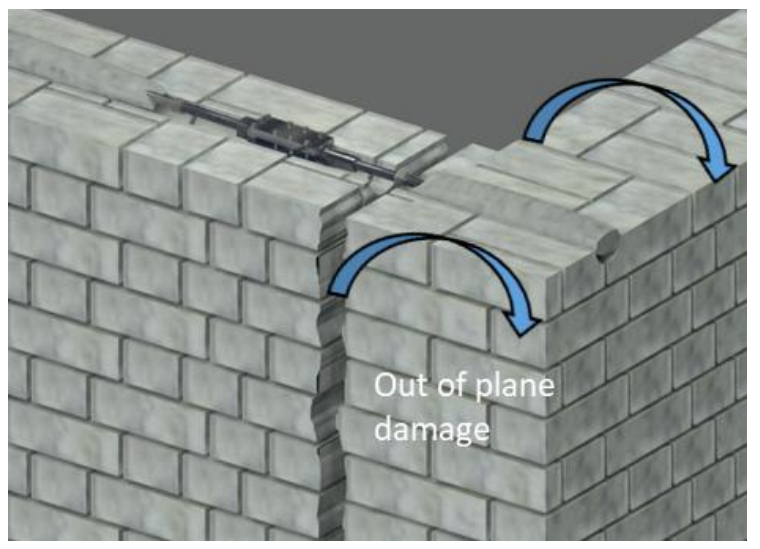

Figure 1: a) Patented design of the dissipative device, b) Insertion of the device at the connection between perpendicular walls (Paganoni 2015)

In the first part of this work, the experimental and numerical activities that led to the development and refinement of the dissipative device are presented. The experimental tests carried out on the device in its isolated configuration allowed for the identification of the meaningful parameters governing the functioning of the device. The dissipative anchoring system was then connected to a brickwork masonry specimen and tested under pseudo-static monotonic loading (pull- out test). The experimental activity demonstrate the beneficial effects of the dissipative anchoring system with respect to his strength-only counterpart.

Drawing on the experimental results, FE models were implemented using the commercial software Abaqus. The numerical simulations led to the refinement of the device's design to obtain a stable and repeatable behaviour. Moreover, the model allows simulating the response of the innovative system to a range of simulated inputs and provides information about the distribution of stresses both in the device and in the masonry wall.

A number of parameters have been analysed to determine which give the best results in terms of computational expediency and accuracy in respect to the behaviour of the laboratory samples.

In the second part of this work, the identified parameters feed in the model of a structure that is used to study the effect of standard and dissipative anchors ties on a larger scale. The building geometry provides a suitable case study as the analyses showed that it could be affected by local/global overturning damage of the walls in case of a seismic event. The model is used to predict the structural response in different scenarios, to guide future experimental activities and to fulfil the computational validation of the dissipative devices with respect to future applications to real case buildings.

\section{THE FRICTION-BASED DISSIPATIVE ANCHORING SYSTEM}

\subsection{Optimization of the friction-based dissipative device}

The design of the friction device draws on the Coulomb equation of friction:

$$
F_{/ /}=n \mu F_{\perp}
$$


Where $F_{\perp}$, the perpendicular force, represents the force transferred from the external plates to the internal slider by tightening two lines of bolts that pass through the assembly as shown in Figure 2. The friction force resisting the sliding motion is the combination of the perpendicular force, the friction coefficient, $\mu$, and the number of surfaces in contact, $\mathrm{n}$. The relative motion between the slider and the plates is initiated when the force acting in their own plane, $F_{/ /}$, exceeds the static friction force. Finally, a steel pin inserted in the device limits the sliding run of the internal part. Figure 2 shows in detail the elements composing the final design and the way the bolt load is transferred.
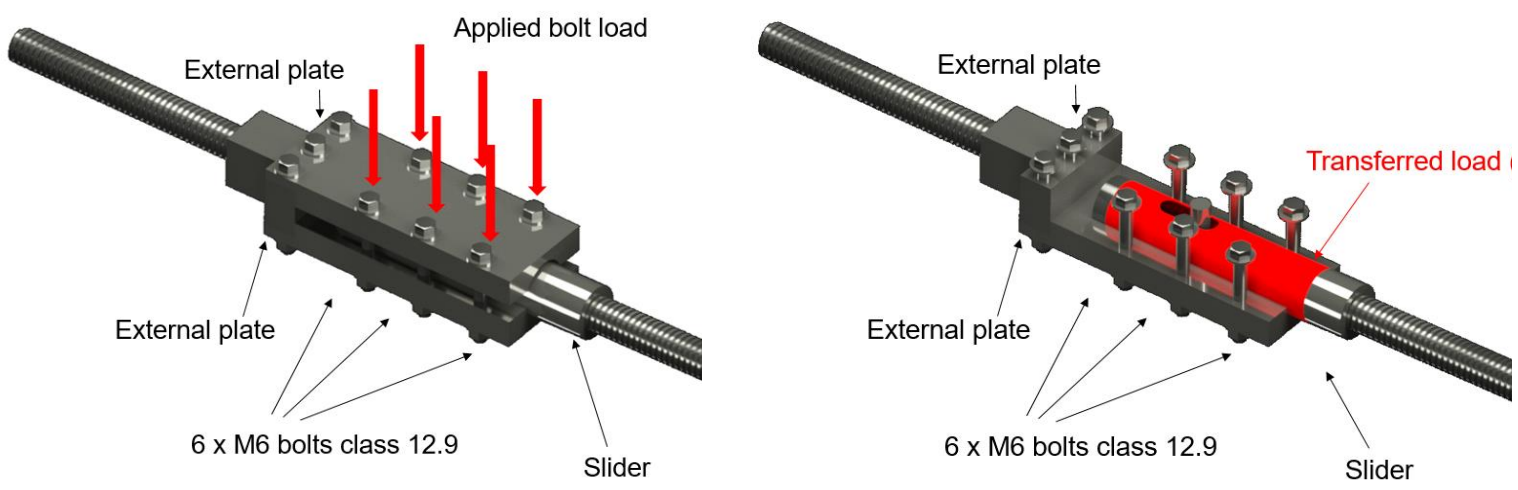

Figure 2: In friction-based device the force is transferred to the internal slider by tightening two lines of bolts

The validation process of the dissipative anchoring system is carried out through experimental and computational activities. Starting from the friction-based device developed by D'Ayala and Paganoni within the partnership between Cintec International and the University of Bath [13], the design was refined on the basis of experimental results conducted at University College London under a new collaboration agreement [14]. The fatigue tests conducted on the initial prototype had shown that the ratio between slip load and applied perpendicular force $(\Phi)$ significantly increases with the number of cycles. This variation is mainly caused by the wearing of the contact surfaces, which led to cold welding and interlocking phenomenon. Therefore, a detailed 3D FE model was developed in Abaqus FEA 6.14 to achieve a more stable and reliable behaviour under cyclic loading. From the analysis of the stress distribution, it was concluded that a cylindrical shape for the sliding leads to a more homogeneous distribution of the shear stresses in the contact area. Figure 3 reports this evidence showing the larger contact area achieved by a cylindrical slider with respect to a squared one [14].

a)

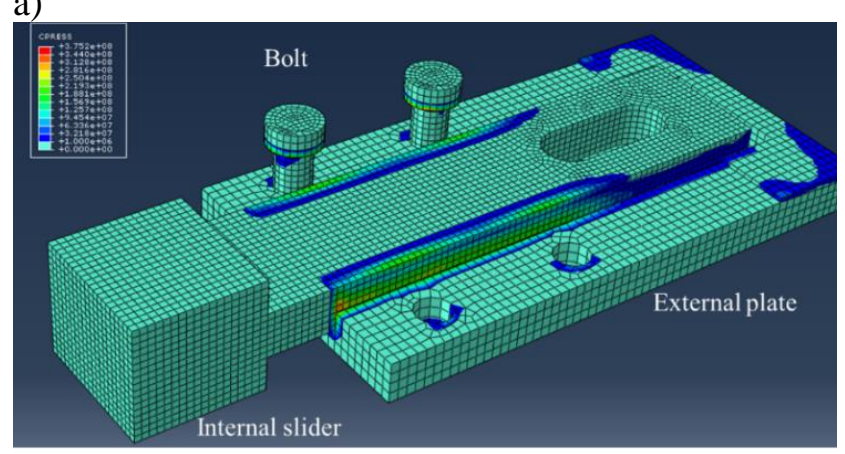

b)

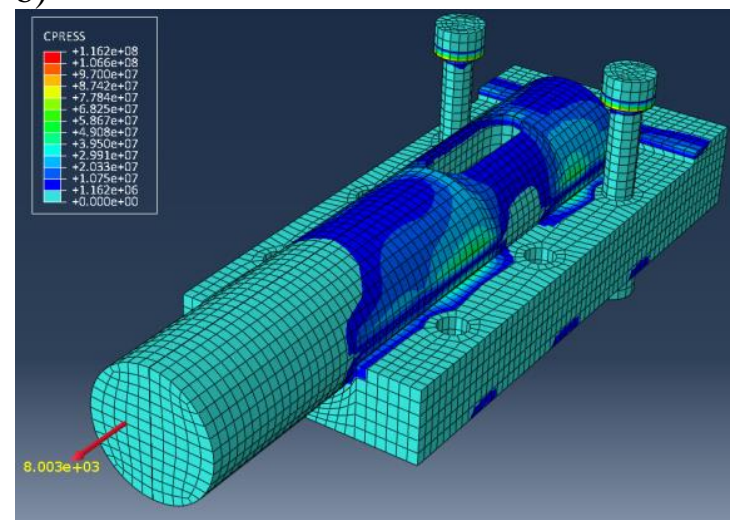

Figure 3: Numerical model: contact area a) of the initial prototype featuring a squared slider and b) of revised prototype featuring a cylindrical slider 


\subsection{Monotonic pull-out tests - Experimental and numerical analysis}

A second experimental campaign aims at evaluating the behaviour of the dissipative device connected in series to a stainless steel bar grouted into a masonry panel. To this purpose a set of pull-out tests were performed to analyse the behaviour of a connection between two vertical elements, i.e. walls, strengthened by the dissipative anchoring system. The focus is on the performance of the anchor, and the damage caused to the wall perpendicular to the anchoring direction by the monotonic action of pull-out. The test set-up consists of the portion of stainless steel anchor embedded in a masonry panel perpendicular to the main seismic action, while the pulling action of the testing apparatus simulates the reaction of the anchor lying within the wall parallel to the main shock direction. Finally, the device is installed as add-on element between the grouted anchor and the pull-out jack as shown in Figure 4.

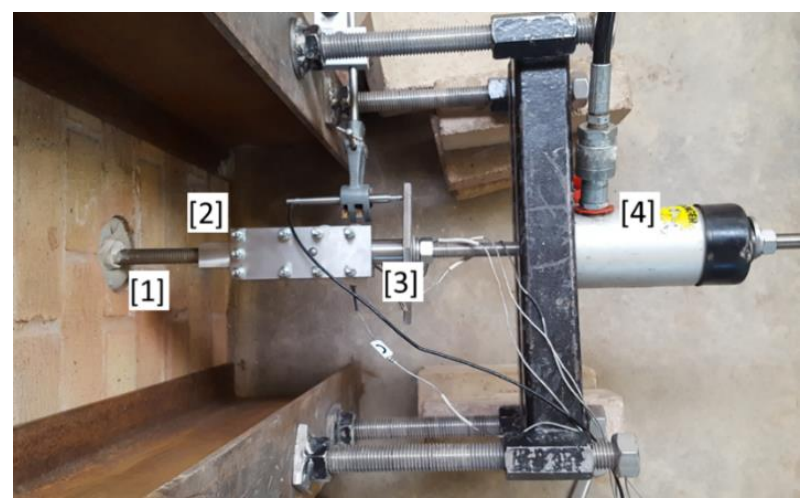

Figure 4: Description of elements composing the testing set up.

After visual inspection of the masonry panel, it was concluded that the devices can address some of the drawbacks that are identified for standard anchors, such as the presence of damage in the substratum, even for small relative displacement between the grouted anchor and the masonry. Conversely, the friction device improves the response of anchors, considerably reducing, or eliminating damage to the parent material. The activation load that initiates its sliding motion can be tuned to the point that almost no relative displacement is detected in the other elements of the anchor assembly.

A Finite Element model is implemented in Abaqus FEA 6.14 to understand the interaction between parent material and anchoring system. This is a central task of the validation process as the bond between the masonry and the grout determines the load at which the failure mode occurs. A numerical model of the dissipative device connected to a steel bar embedded in a masonry wall was generated, as shown in Figure 5.

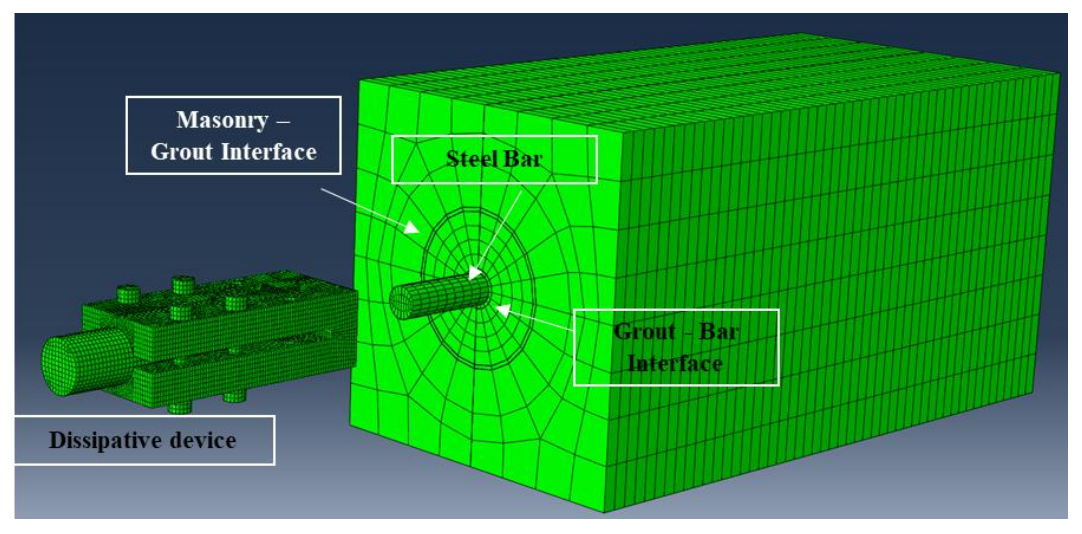

Figure 5: Numerical model - Undeformed shape and components of the model. 
Three-dimensional volume elements were used for the mesh since an accurate stress distribution is relevant for a clear understanding of the stress field and of the structural behaviour. The motion of the steel bar is rigidly coupled to the device's end plate. The mesh discretization was defined to achieve a compromise between accuracy and efficiency. Brick elements (C3D8R, eight-node linear brick with hourglass control) are used for the mesh of all the parts, except for the simulation of the bond between different materials, where tridimensional cohesive elements (COH3D8, eight nodes in each surface) were used. The generated mesh includes 41309 nodes and 31111 elements.

According to the failure mechanism observed in the experimental activity, interface elements around the steel bar (inner interface) and between the grout and the masonry (outer interface) were included. The interfaces aim to simulate the bonding properties among the single components, i.e. the steel bar, the grout and the masonry. Abaqus FEA allows for the selection of cohesive elements to which a linear elastic traction-separation law before damage has been assigned. When the maximum traction resistance is reached, the interface elements display a progressive degradation, which is driven by a damage process. The numerical parameters defining the mechanical properties of these cohesive elements were calibrated to replicate the experimental behaviour of the sample under out-of-plane loading and reproduce the different failure mechanism.

The shear stiffness of the external interface is defined according to the pull-out tests performed on the specimens of anchors embedded in the masonry wall.. In order to define the traction-separation law that describes the behaviour of the cohesive interface, the values of maximum traction resistance $\tau_{\max }$ and ultimate dispacement $\delta_{\text {ult }}$ need to be provided. On the basis of the experimental data, a mean value of $F_{\max }=24 \mathrm{KN}$ was considered to compute the maximum shear resistance $\tau_{\max }$ :

$$
\tau_{\max }=\frac{F_{\max }}{A_{\text {interf }}}=0.29 \mathrm{MPa}
$$

The change in stiffness exhibited by the anchors at a recorded displacement of $0.5 \mathrm{~mm}$ determines the end-point of the linear correlation between the shear stress and the shear deformation. The Shear modulus $(\mathrm{G})$ is therefore equal to:

$$
G=\frac{\tau_{\max }}{\gamma}=\frac{0.29}{0.25}=1.16 \mathrm{MPa}
$$

The pull-out tests performed on the anchor embedded in the masonry wall highlighted that the most likely failure was slippage of the bar-socket assembly, caused by the loss of adherence between the grouted socket and the surrounding material. Therefore, the ultimate displacement refers to the relative displacement of the grout with respect to the masonry at which the anchoring system is no longer able to provide any resistance to the pull-out force. According to the experimental data and crack propagation this value was assumed equal to $\delta_{\text {ult }}=5 \mathrm{~mm}$. The analytical curve implemented in the numerical model shown in Figure 6. 


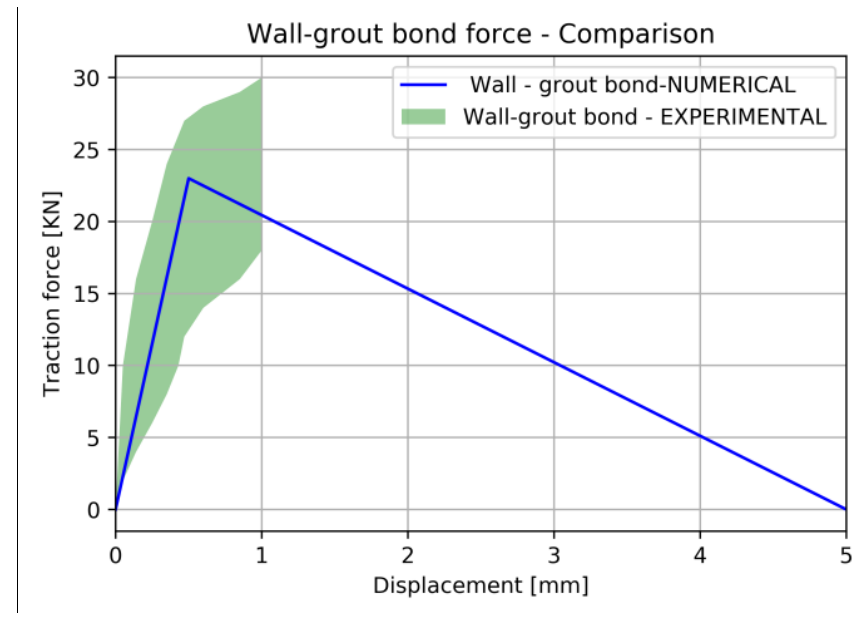

Figure 6: Comparison between the experimental envelope and the numerical analysis

Regarding the physical and mechanical properties of the grout and the steel, information from the technical sheet were used. Table 1 provides the mechanical properties adopted for the materials, as well as the parameters needed to define the traction-separation law for the interfaces.

\begin{tabular}{|c|c|c|c|c|c|}
\hline Part & E & G1 & tmax & displacement at tmax & Ultimate damage \\
\hline & {$[\mathrm{Pa}]$} & {$[\mathrm{Pa}]$} & {$[\mathrm{Pa}]$} & {$[\mathrm{m}]$} & {$[\mathrm{m}]$} \\
\cline { 2 - 6 } Wall & $1.50 \mathrm{E}+09$ & & & & \\
OUT Interface & - & $1.16 \mathrm{E}+06$ & $2.90 \mathrm{E}+05$ & $5.00 \mathrm{E}-04$ & $5.00 \mathrm{E}-03$ \\
INT Interface & - & $2.00 \mathrm{E}+08$ & $5.00 \mathrm{E}+07$ & $5.00 \mathrm{E}-04$ & $5.00 \mathrm{E}-03$ \\
Grout & $3.00 \mathrm{E}+10$ & & & & \\
Steel bar & $2.10 \mathrm{E}+11$ & & & & \\
\hline
\end{tabular}

Table 1: mechanical properties of model materials

The stress field attained in various parts of the model can be visualised through an analysis run setting a maximum displacement of $40 \mathrm{~mm}$ to the internal slider of the device. Figure 7 shows the failure of the outer interface caused by the imposed displacement.
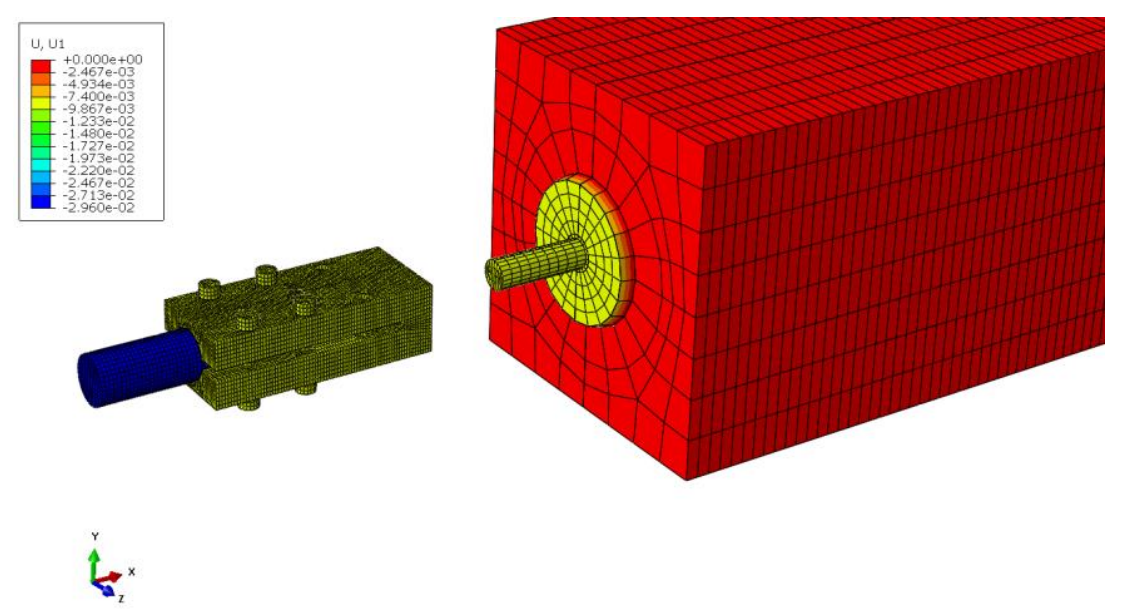

Figure 7: Numerical model - Deformed shape, failure at wall-grout interface.

In conclusion, the FE model of the pull-out test succeeds in replicating the bond failure between the parent material and the grouted element of the standard anchor, this being the failure mode that controls the performance of the whole assembly. The definition of a fictitious 
part located between the grouted socket and the parent material and defined by tractionseparation low is suitable for this purpose. The material properties of the fictitious part are defined on the basis of experimental results, creating an equivalent stress-strain curve compatible with the chosen failure criterion. Therefore, the numerical model tuned on the obtained experimental results can be considered good approximation of the physical test and can be used as a valid tool for the optimization of an upgrade intervention. Drawing on these conclusions, a second numerical model was developed to investigate the structural behaviour of a portion of an idealized masonry building under seismic loading.

\section{FE MODELS OF AN IDEALIZED CASE-STUDY STRUCTURE}

The positive outcome of the model of the pull-outs means that the modelling technique of fictitious parts and equivalent material curves can be applied to numerically describe the bonding capacity of a grouted anchor. The parameters identified for the pull-out model are applied for the model of a structure that is used to study the effect of standard and dissipative anchoring system on a larger scale. The analysed structure is composed of a front wall connected to two sidewalls representing an idealized portion of a masonry building with poor quality connection between adjacent walls. The geometry and dimensions of the subassembly are shown in Figure 8.

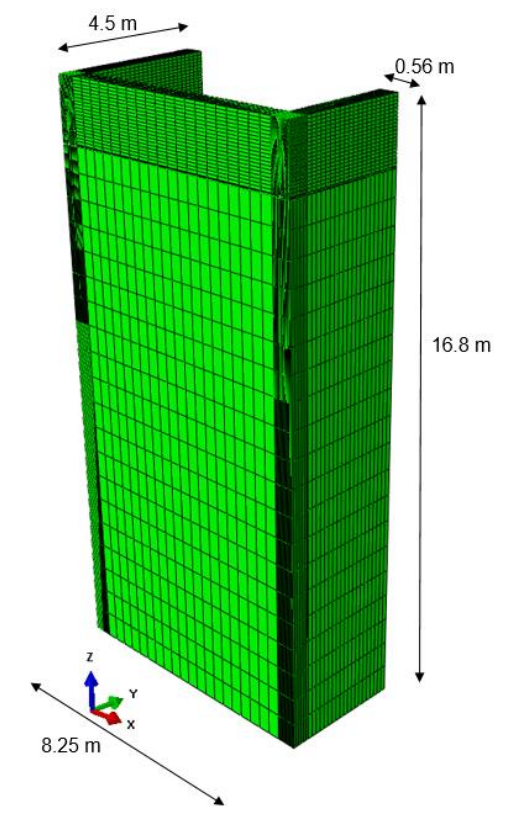

Figure 8: a) Dimensions of the analyzed corner connection

Modelling a smaller portion of the building allows for a more detailed analysis of the behaviour of the connection both in the unstrengthened and strengthened configuration. Three FE models are implemented: a first model represents the original structure, where the connection between the adjacent walls relies on their shear capacity only. A second model represents the structure when traditional grouted ties are inserted at the corner connection. In a last model, a friction-based dissipative device is implemented in series with the strength-only anchors to provide the connection with energy dissipation capacity. The response of the structure is evaluated imposing a seismic base acceleration in the out of plane direction of the front wall. The ground acceleration of the 2009 L'Aquila earthquake (Mw 6.3 main shock), as recorded at the station of L'Aquila - Valle Aterno (station code AQV) is used as input for the analysis. The walls are defined assuming that the mortar joints and brick units are smeared into 
one isotropic material, with average mechanical properties according to the principle of macromodelling [15].

\subsection{The original structure}

In the first model, the connection between cross walls relies on the equivalent traction resistance of a set of interfaces. An equivalent traction capacity is assigned to these interfaces in the direction of their thickness $(\mathrm{T}=0.002 \mathrm{~m})$. This allows for the modelling of the shear resistance that opposes the out-of-plane loading that causes the wall's separation. If the external load exceeds the shear force the detachment of the adjacent walls is no longer prevented and the front wall experiences outward tilting. A traction-separation law is assigned to describe the mechanical constitutive behaviour of the interface. According to the Mohr-Coulomb criterion, the linear relationship between the shear strength $\tau$ and the normal compressive stress $\sigma$ is given by the formula:

$$
\tau=c+\sigma \tan (\varphi)
$$

Where $\mathrm{c}$ denotes the cohesion and $\varphi$ the angle of internal friction. For this study, the average cohesion and friction angle in the bed joints are respectively $0.35 \mathrm{MPa}$ and $0.5 \mathrm{rad}$. The equivalent traction force ( $F_{\text {traction }}$ ) is therefore the integral of the shear stress over the interface's area:

$$
\begin{gathered}
F_{\text {traction }}=\int \tau d A=\tau \cdot A_{\text {interface }} \\
\sigma_{\text {traction }}=\frac{F_{\text {traction }}}{A_{\text {interface }}}=\tau
\end{gathered}
$$

Therefore for the purpose of this model the traction tress $(\sigma)$ is equivalent to the the shear stress $(\tau)$. The shear stress linearly increases with the compressive stress, which means that the interfaces have higher equivalent traction resistance moving from the top to the bottom of the wall. The graph Figure $9 \mathrm{~b}$ shows the values of equivalent traction resistance according to the location of the interfaces as measured from a control point (CP), referenced in Figure 9. To consider the damage evolution a post-damage effective separation at failure is defined and a coefficient of friction $(\mu)$ is specified to prevent components' penetration. Figure 9 a shows the location of the upper cohesive interface with respect to the side and front walls.

a)

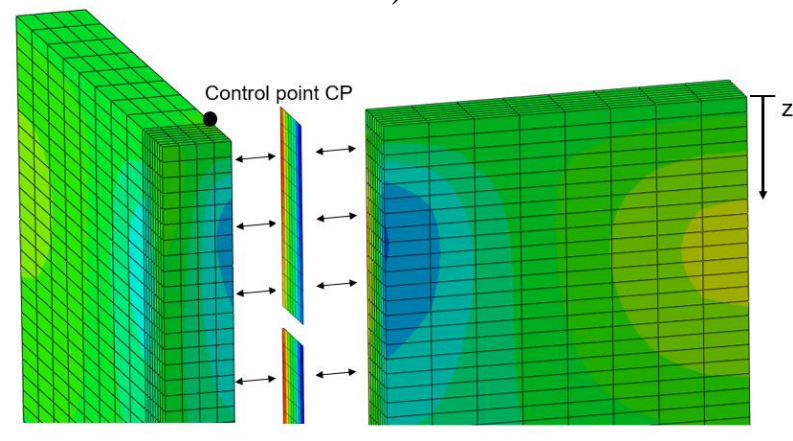

b)

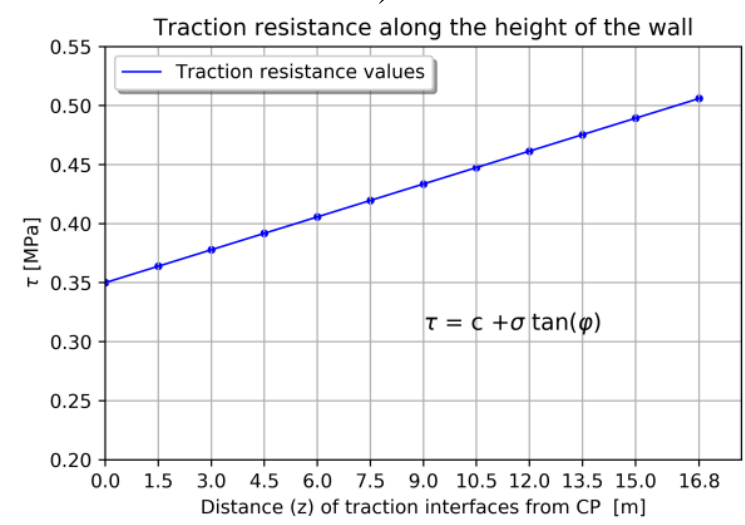

Figure 9: Model of unstrengthened walls: a) location of interface part with respect to walls b) Traction resistance of the cohesive interfaces along with the height of the wall. 
As a result of the applied base acceleration shown in Figure 11a, the orthogonal walls separate when the strain in the direction of the interfaces' thickness $(\mathrm{T})$ exceed the maximum strains defined by the traction-separation low. The strain limits depend on the maximum traction stress $\sigma_{\max }$ assigned to the interface according to Equation 4 . The computation carried out to determine the ultimate shear resistance for the upper interface are:

$$
\begin{gathered}
F=K \Delta u=\frac{E A}{T} \Delta u \\
\sigma_{\text {traction }}=\frac{E}{T} \Delta u=\frac{E}{0.002} \Delta u=E \varepsilon \\
\varepsilon_{\text {elastic }}=\frac{\sigma_{\text {traction }}}{E}=\frac{3.510^{6}}{3.010^{6}}=0.116=11 \% \\
\varepsilon_{\text {ultimate }}=\frac{\Delta u_{\max }}{T}=\frac{0.005}{0.002}=2.5=250 \%
\end{gathered}
$$

Where $\mathrm{E}$ and $\mathrm{A}$ are the Young modulus and area of the interface, respectively and the maximum elastic strain $\left(\varepsilon_{\text {elastic }}\right)$ and ultimate strain $\left(\varepsilon_{\text {ultimate }}\right)$ are expressed as a percentage of the initial thickness of the interface $(\mathrm{T}=2 \mathrm{~mm})$. The resulting constitutive laws assigned to the cohesive interface at $\mathrm{z}=0 \mathrm{~m}$ (upper interface) and the cohesive interface at $\mathrm{z}=15 \mathrm{~m}$ (lower interface) are shown in Figure 10a.
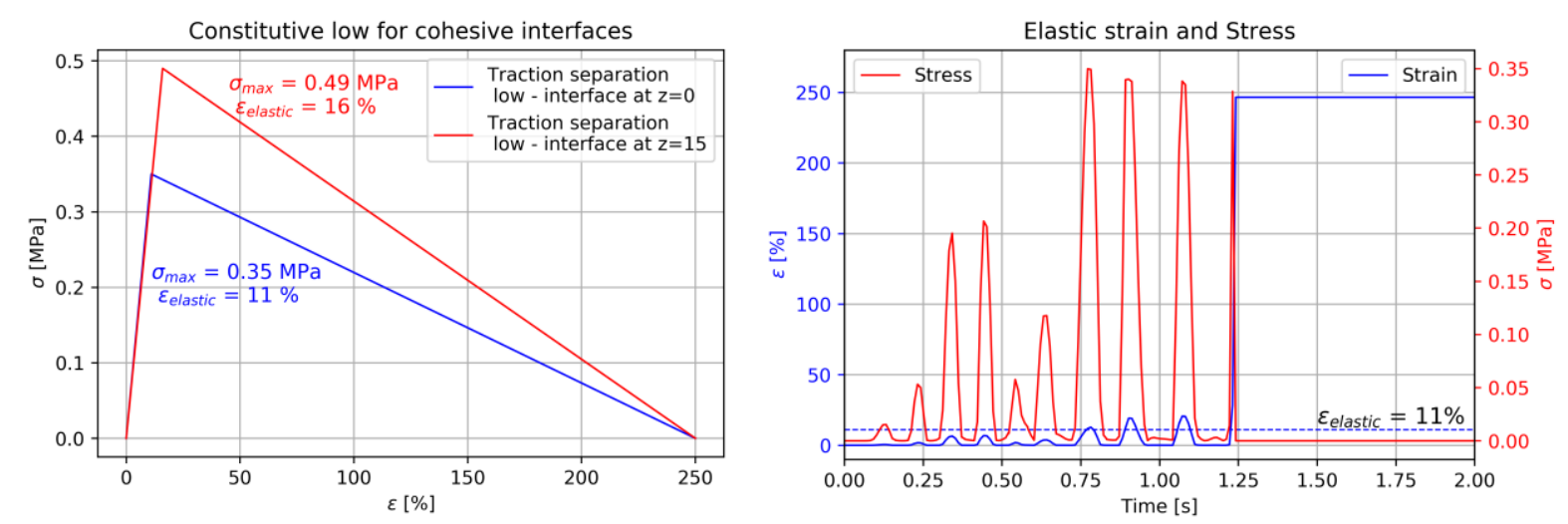

Figure 10: a) Constitutive law for the cohesive interface, b) strain and stress field for the upper cohesive interface

The graph reported in Figure 10b shows that the seismic acceleration applied to the base of the model produces in the upper interface a strain field equal to $11 \%$ and $250 \%$ after $0.76 \mathrm{~s}$ and $1.25 \mathrm{~s}$ respectively. For these values of strain, the interface elements experience the maximum values of traction stress $(0.35 \mathrm{MPa})$ and the failure. Once the damage is initiated, it propagates down the height of the wall and the orthogonal walls start oscillating independently. The CP experiences a maximum relative displacement from the sidewall of $0.35 \mathrm{~m}$ at time step $1.25 \mathrm{~s}$ and the height of the separation is 6.8 meters as shown in Figure 11. 

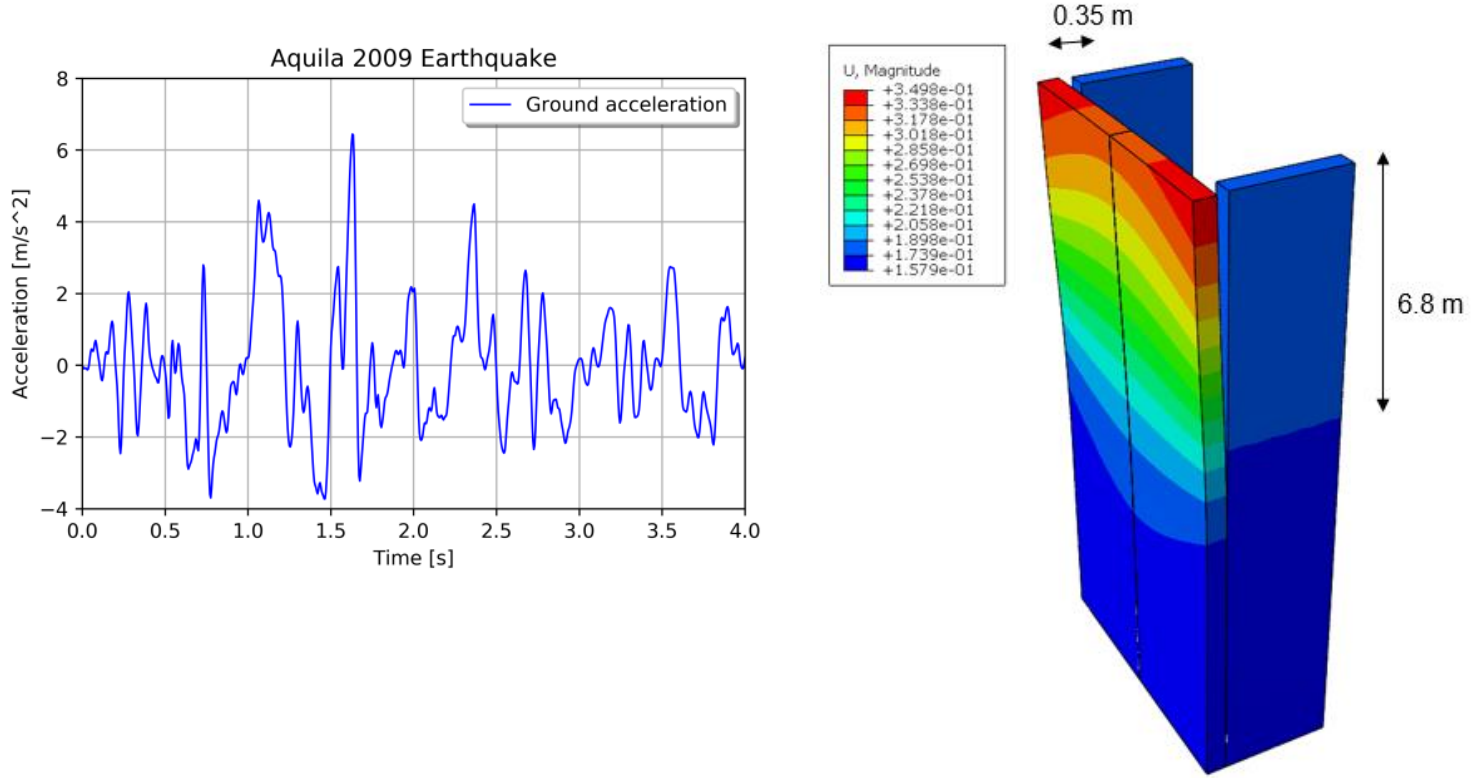

Figure 11: a) Applied base acceleration, b) deformed shape of FE model at time step 1.25 seconds.

These results show that the FE model is able to reproduce the typical failure mechanism detected on site in the aftermath of a seismic event, when major cracks at the interface between cross walls are often observed. The analysis shows that the connection capacity simulated by the interfaces is not sufficient to restrain the walls. The lack of effective lateral constrains determines the façade detachment form the side walls and it deforms similarly to a cantilever beam undergoing bending, as shown in Figure 11b. The model is deemed suitable for predicting the response of the structure in other case scenarios, for instance by inserting the anchors and dissipative devices characterised in section 2.2.

\subsection{The strengthened structure}

A second model is implemented to investigate the benefit of introducing a reinforcement scheme able to improve the connection between the cross walls that experienced mutual separation. Damage experienced by the structure is accounted for by removing the interfaces for the extent they failed in the previous analysis.

Material properties are the same as for the undamaged model, as cracking is localised and does not affect other parts of the structure; thus, it is not necessary to reduce the stiffness of the parent material. Table 2 provides the mechanical properties adopted for the materials.

\begin{tabular}{|c|c|c|c|c|c|c|c|}
\hline Part & E & G1 & G2 & Density & Maxs Damage & First Damage & Ultimate damage \\
\hline \multirow{4}{*}{ Wall } & {$[\mathrm{Pa}]$} & {$[\mathrm{Pa}]$} & {$[\mathrm{Pa}]$} & & normal[Pa] & {$[\mathrm{m}]$} & {$[\mathrm{m}]$} \\
\cline { 2 - 8 } & $1.50 \mathrm{E}+09$ & & & $2.14 \mathrm{E}+03$ & & & \\
Interface & $3.00 \mathrm{E}+06$ & $3.00 \mathrm{E}+06$ & $3.00 \mathrm{E}+06$ & $1.75 \mathrm{E}+03$ & $3.50 \mathrm{E}+05$ & $2.33 \mathrm{E}-04$ & $5.00 \mathrm{E}-03$ \\
Steel bar & $2.10 \mathrm{E}+11$ & & & $8.00 \mathrm{E}+03$ & & & \\
\hline
\end{tabular}

Table 2: mechanical properties of model's materials

A first FE analysis is performed on a model including standard stainless steel ties. A common retrofit practice is to grout the ties within the masonry inside a drilled hole to restore the boxlike behaviour of masonry structures. Therefore, in the numerical model the metallic elements run along the whole length of the side walls, reconnecting the sets of perpendicular walls. 
Paganoni and D'Ayala [16] used mono-dimensional truss elements to model the ties and a bonded contact was implemented at the interface between truss and elements representing the masonry. In the present work the anchors are modelled by means of three-dimensional elements and an interface part is defined to govern the mutual behaviour between the anchor and the parent material. The insertion of the grouted steel anchors is modelled through cohesive interfaces which reproduce the bonding between the ties and the masonry. A traction-separation law with sub-options governing the post damage behaviour is assigned to the cohesive elements. The parts representing the front and side walls are rigidly connected for 10 meters from the base by numerically defining tie constrains which prevent the relative motion of nodes belonging to the side and front wall. The two parts are disconnected for the remaining 6.8 meters and a frictionless contact coefficient $(\mu)$ is specified to prevent components' penetration. This means that, for this portion of the wall, the only link preventing the relative motion of the orthogonal walls is provided by the anchors running through the two parts representing the walls.

Compared to the model without any strengthening solution, the insertion of metallic ties does not improve the overall behaviour of the structure. The interface controlling the bonding capacity between the anchor and the surrounding part reaches the maximum traction capacity after 0.18 seconds and the anchor is pulled out of the front wall after 0.51 seconds when the interface experiences the maximum allowable displacement, namely $5 \mathrm{~mm}$. The pull-out failure does not extend to the whole length of the anchor, but affects instead the portion adjacent to the disconnection, i.e. the portion bridging the vertical crack between front and side wall. Before failure, the maximum stress concentrations are registered in the proximity of the anchorage point on the façade face, as shown in Figure 13. The model shows a punching/pull-out failure at the head of the anchorage, which is a recurring failure mode for this type of retrofitting system in case of weak substratum, as shown in Figure 12.

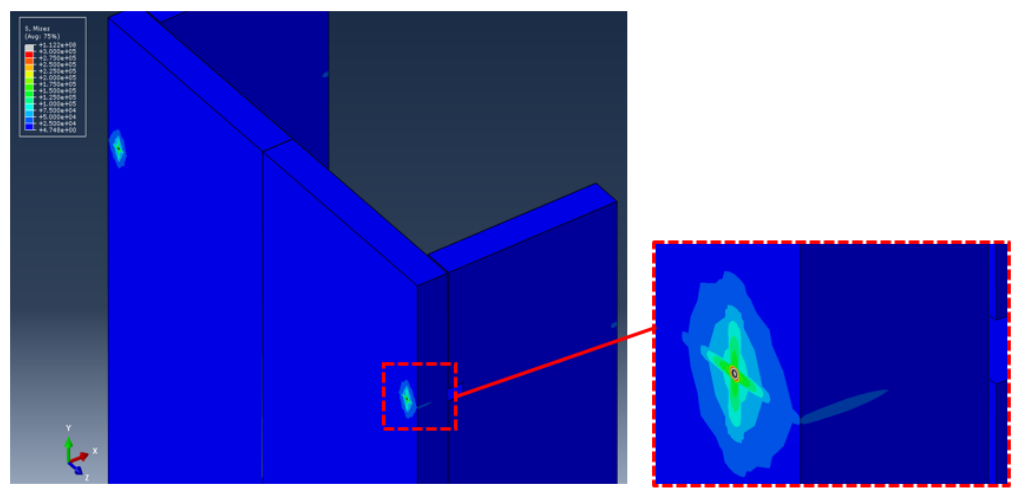

Figure 12: Punching failure of standard metallic ties - FEM

To observe in detail the stress field in the material surrounding the anchorage, the parts composing the model are sectioned along the $\mathrm{x}-\mathrm{z}$ plane where the longitudinal axis of the anchor lies. Figure 13 shows the stress distribution along the length of the interface. As the front wall tilts outwards, the part representing the grouted bars are bent along their longitudinal axis. This mechanism determines that the maximum tensions are equal in value but opposite in sign as shown in Figure 13. Two critical points in the time history are identified, namely the time steps when the maximum traction and the maximum displacement are reached. 


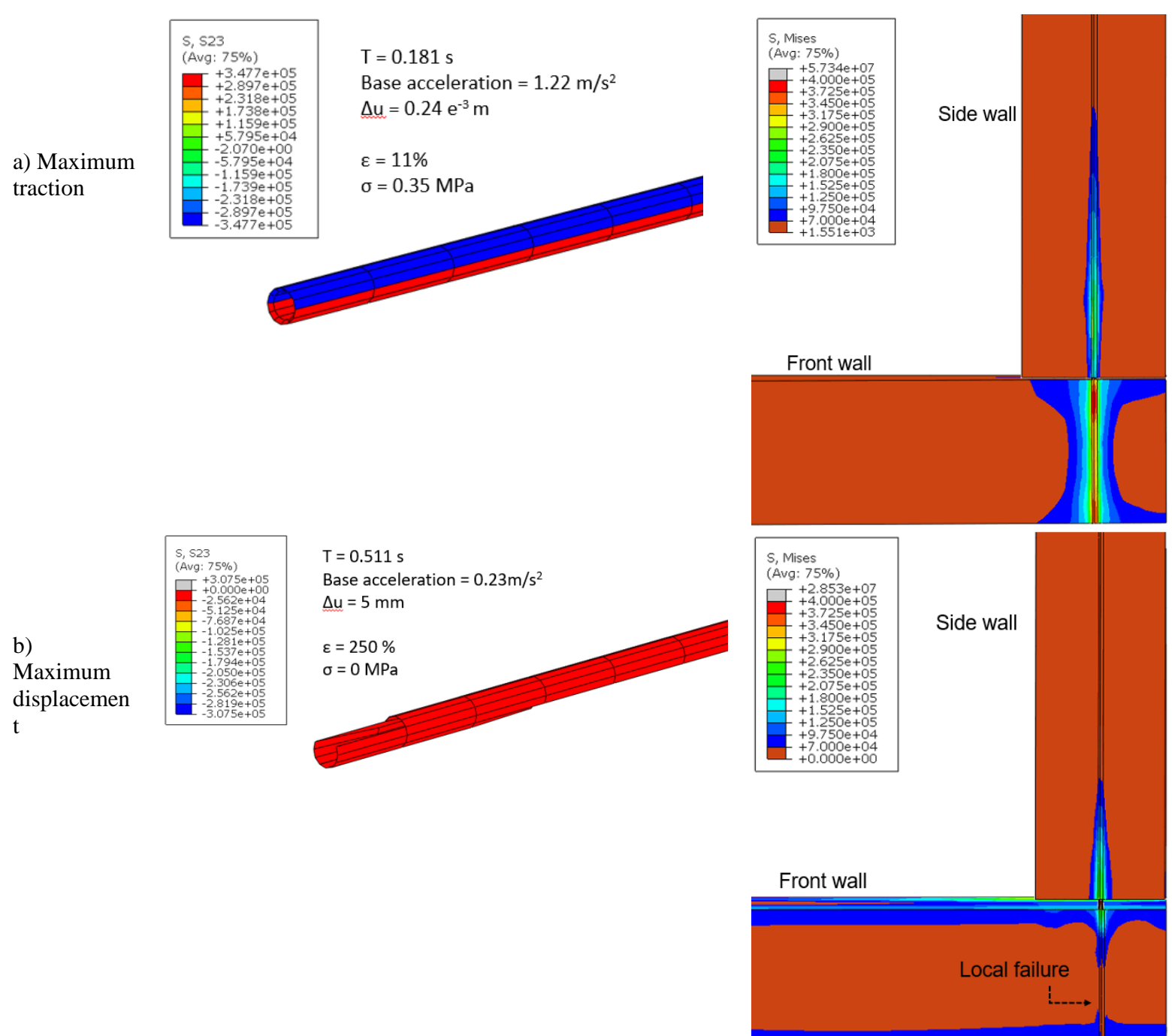

Figure 13: Distribution of Shear stresses along the cohesive interface and V. Mises stresses as transferred from the anchors to the masonry walls

A second model including a friction-based device inserted at the joint between front and side walls undergoes the same input as the model with standard steel ties. The dissipative device is modelled with solid elements reproducing all the parts composing the final assembly and is connected rigidly to the steel bars. The friction force resisting the slider's motion is induced by defining a "bolt load" in the element reproducing the bolts of the real devices. Each bolt-part is loaded with a transversal force equal to $2700 \mathrm{~N}$ and the resulting friction force is computed according to Equation 1:

$$
F_{/ /}=n \mu F_{\perp}=4 \cdot 0.2 \cdot(4 \cdot 2700)=8.6 \mathrm{KN}
$$

As for the previous model, one cohesive interface connecting the bars to the surrounding elements complete the assembly. Figure 14a shows the sliding motion of the device's slider along with the acceleration experienced at the anchor's location, namely at 14 meters from the base. From the graph, it is clear that the device activates twice depending on the experienced acceleration: the first time at $\mathrm{T}=0.26$, when it oscillates between its initial position and $2.5 \mathrm{~mm}$ and the second time at $\mathrm{T}=1.16 \mathrm{~s}$ when it fully exploits the allowable run of $20 \mathrm{~mm}$. Since the device is tuned to start sliding for a resulting force $(8.6 \mathrm{KN})$ lower than the one that would 
cause the pull-out failure of the assembly $(11 \mathrm{KN})$ the stress distribution in the wall's part are lower than the maximum traction capacity.

a)

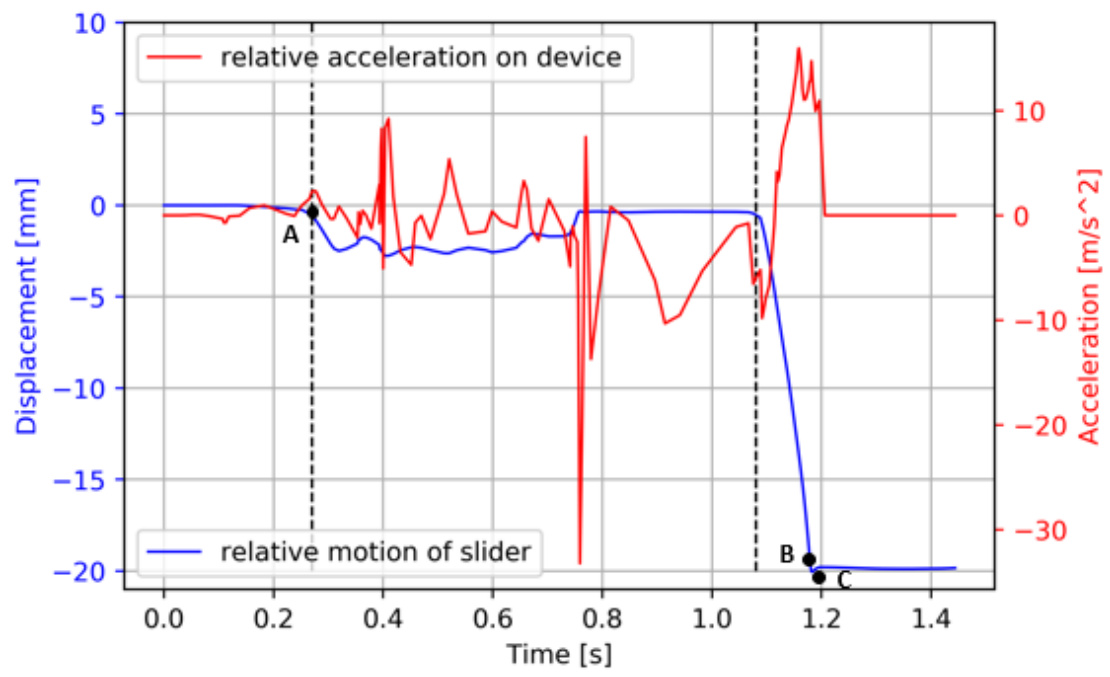

b)

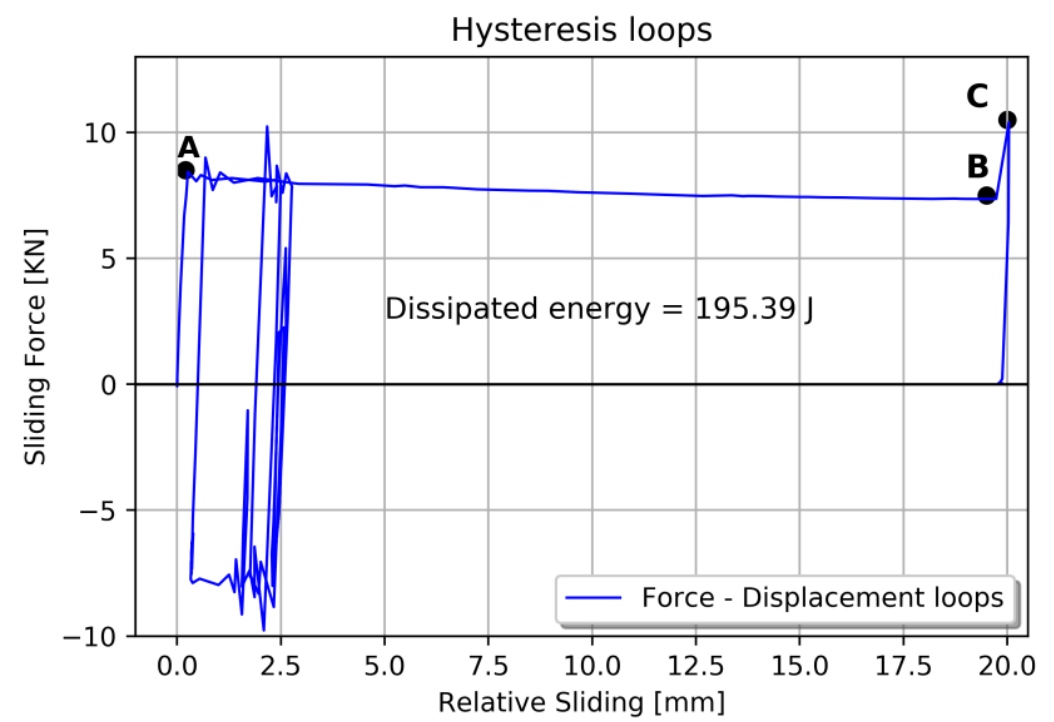

Figure 14: Sliding motion of the internal part and relative acceleration at anchor's location, b) Hysteresis loops of friction-based dissipative device

Figure 14b shows the load-displacement curve experienced by the device. When the sliding force exceeds the friction resistance force (Point A), the sliding central part starts moving according to the direction of the applied base acceleration. In particular, the slider completes a hysteresis loop oscillating between its initial position and $2.5 \mathrm{~mm}$. At $\mathrm{T}=1.16 \mathrm{~s}$, it slides out for $20 \mathrm{~mm}$, thus reaching the maximum controlled displacement of the device (Point B). Point C in Figure 14 represents the ultimate traction resistance that the interface around the steel bar part can provide, after which the interface displays a gradual degradation. The areas between the force-displacement curve and the $\mathrm{x}$-axis represents the total energy that the system can provide to resist the applied motion.

Figure 15 shows the stress distribution at the time step corresponding to the maximum traction and maximum displacement in the interface part. At time $\mathrm{T}=1.18 \mathrm{~s}$ the frictional sliding occurring in the device is equal to $20 \mathrm{~mm}$ and the internal part hits the central pin that controls 
the allowed motion of the slider. At time $\mathrm{T}=1.20 \mathrm{~s}$ the system, now working as a traditional strength-only anchor, fails as the interface between the bar and the masonry has reached the maximum displacement, namely $5 \mathrm{~mm}$. By comparing the stress distribution in the parts surrounding the anchors, it can be noted that the insertion of the dissipative device reduces the shear stresses transferred from the anchor to the walls.

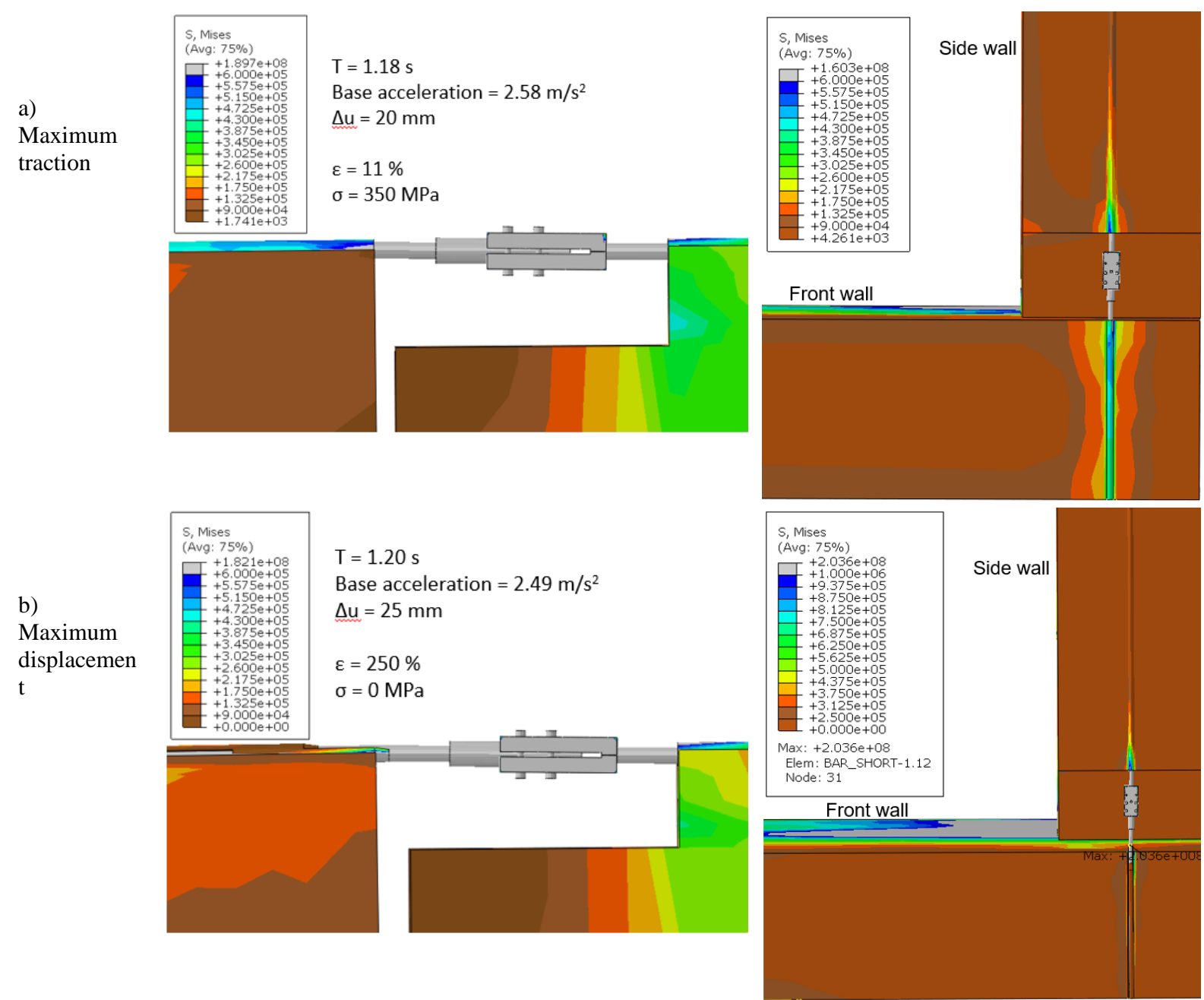

Figure 15: FE model of a corner connection strengthened by means of dissipative anchoring system. Deformed shape and stress distribution at maximum traction and maximum displacement

Figure 16a shows the relative acceleration experienced by the orthogonal walls at $14 \mathrm{~m}$ from the base, namely where the strengthening systems are inserted. From the graph, it is clear that the corner connection strengthened by means of the dissipative device withstands the applied base acceleration for a longer period. The ultimate failure is reached at $\mathrm{T}=1.2 \mathrm{~s}$, whilst it was reached at $\mathrm{T}=0.5 \mathrm{~s}$ for the traditional anchor. 
a)

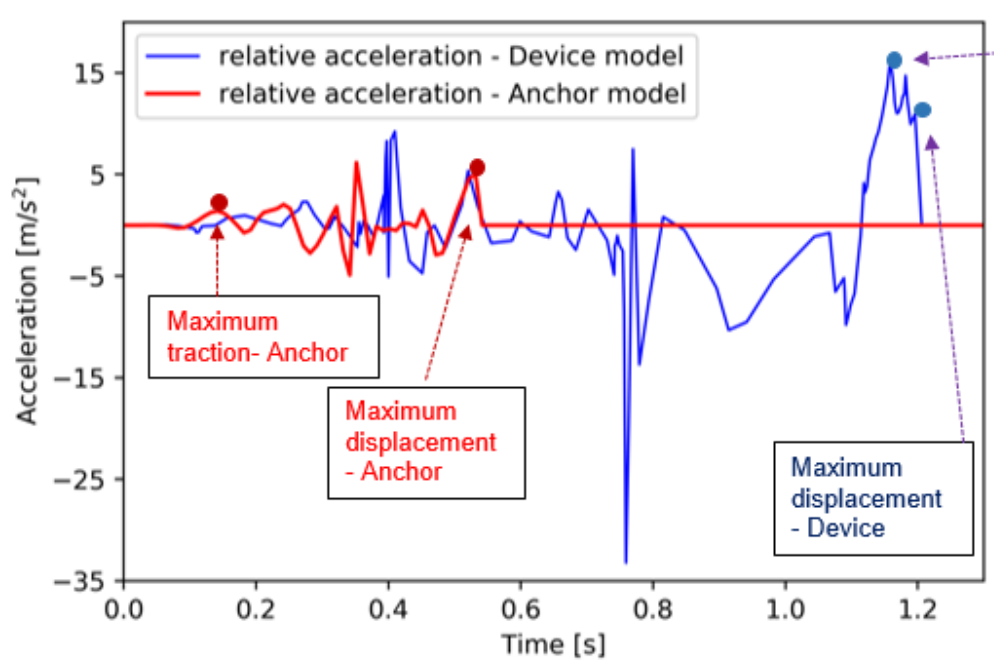

Maximum

traction- Device

b)

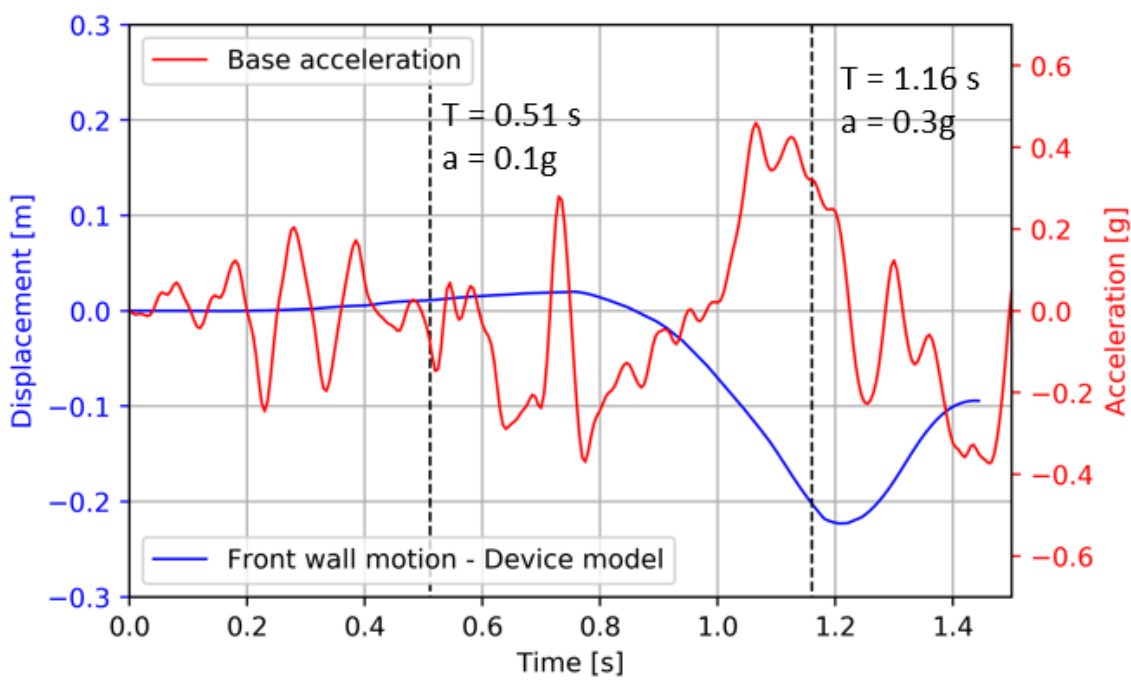

Figure 16: a) Relative acceleration experienced by the strengthening systems, b) base acceleration and displacement time history of front wall

By comparing the time steps at which the ultimate failure takes place, Figure 16b shows that the dissipative anchoring system experiences a pull-out failure for a base acceleration equal to $0.3 \mathrm{~g}$ while the model representing the traditional strengthening system fails for a base acceleration equal to $0.1 \mathrm{~g}$.

\section{CONCLUSIONS}

The lack of effective connections between perpendicular walls often determines the failure of a historic masonry structure under seismic loading. To restore the box-like behaviour and provide these particular buildings with sufficient ductility to withstand shaking, the authors developed a friction-based dissipative anchoring system.

Such system comprises a device installed in series with standard metallic anchors grouted at the joint between perpendicular sets of walls of historic structures. The purpose of the strengthening system is to reconnect structural elements that might have become detached as 
result of seismic events, but also to control relative displacements and reduce accelerations in case of further earthquakes, so that damage to the precious wall finishes can be reduced.

The device provides ductility to the connection, mitigating the width of the cracks in the parent material and preventing fragile failures, such as pull-outs. This, of course, entails allowing a controlled out-of-plane deformation, rather than completely relying on the in-plane stiffness of side walls.

The paper presents the experimental and computational work carried out to assess the performance of the dissipative anchoring system and its applicability to an idealized case study.

A series of FE models were implemented using the commercial software Abaqus to refine the device's shape and optimize its design in terms of stress distribution, stability and reliability

The pull-out tests performed on the device connected to steel bars and embedded in a masonry panel confirmed that the innovative system is effective in reducing the damage propagation to the subtratum in respect to its strength-only counterpart. The experimental results feed in the calibration of a computational model, which reproduces the experimental set up in order to understand better the rules that governs the behaviour of the dissipative anchoring system. A model of a wall panel strengthened by the anchoring devices was created and run and the FE model succeeds in replicating the bond failure between the parent material and the grouted element of the standard anchor, this being the failure mode that controls the performance of the whole assembly. Therefore, the numerical model tuned on the obtained experimental results was used as a starting point to develop a numerical model of an idealized case-study building in need of seismic upgrade. The numerical model reproduces the behaviour of a three-panel corner connection when a seismic base acceleration is applied. The front panel disconnects from the sidewalls for a hight of 6.8 meters and undergo out-of-plane motion. The outward tilting of walls is a recurring mode of failure for historic masonry structures, and the proposed system aims at controlling the opening of cracks and taking advantage of. The model of the corner connection is therefore "strengthened" by implementing an anchoring system with and without the addition of the dissipative device. Fictitious interfaces were used to define the bonding capacity between the parts representing the steel bars and the masonry. This method allows for the definition of an equivalent stress-strain curve compatible with the available experimental results. The dissipative anchoring system experiences a pull-out failure for a base acceleration equal to $0.3 \mathrm{~g}$ while the model representing the traditional strengthening system fails for a base acceleration equal to $0.1 \mathrm{~g}$.

It should be noted that the applied base acceleration caused the pull-out failure of both strengthening systems meaning that the full scale signal was too demanding for traction capacity of the interfaces. Therefore, a future set of analysis will include a parametric study investigating the strengthening systems for smaller range of accelerations. Moreover, in the proposed work only one system was inserted in each corner connection, whilst in a real-case application several devices should be included. Therefore, the reported results will feed into a more complex model where a number of dissipative anchoring system will restrain simultaneously the out-of-plane motion of the front wall.

\section{REFERENCES}

[1] S. Zhu and Y. Zhang. 2014. "Seismic Behaviour of Self-Centring Braced Frame Buildings with Reusable Hysteretic Damping Brace." (February):1329-46. 
[2] Christopoulos, C., R. Tremblay, H. Kim, and M. Lacerte. 2008. "Self-Centering Energy Dissipative Bracing System for the Seismic Resistance of Structures : Development and Validation." 134(January):96-107.

[3] Lagomarsino, Sergio. 2012. “Damage Assessment of Churches after L' Aquila Earthquake ( 2009 )." 73-92.

[4] Wilkinson, Sean et al. 2013. "Observations and Implications of Damage from the Magnitude Mw 6.3 Christchurch, New Zealand Earthquake of 22 February 2011." Bulletin of Earthquake Engineering 11(1):107-40.

[5] Paganoni, Sara and Dina D'Ayala. 2014. "Testing and Design Procedure for Corner Connections of Masonry Heritage Buildings Strengthened by Metallic Grouted Anchors." Engineering Structures 70:278-93.

[6] D'Ayala, Dina F. and Sara Paganoni. 2011. "Assessment and Analysis of Damage in L'Aquila Historic City Centre after 6th April 2009." Bulletin of Earthquake Engineering 9(1):81-104.

[7] 1998:2005, EN. 2011a. "Eurocode 8: Design of Structures for Earthquake Resistance. Part 1 : General Rules, Seismic Actions and Rules for Buildings.” 1(2004).

[8] ICOMOS. 2003. "Principles for the Analysis, Conservation and Structural Restoration of Architectural Heritage ( 2003 )." Architectural Heritage 3-6.

[9] D.P.G.M. 2011. “Linee Guida per La Valutazione e La Riduzione Del Rischio Sismico Del Patrimonio Culturale Con Riferimento Alle Norme Tecniche per Le Costruzioni Di Cui Al Decreto Del Ministero Delle Infrastrutture e Dei Trasporti Del 14 Gennaio 2008 (09/02/2011)." 1(c):1-83.

[10] Indirli, Maurizio and Maria Gabriella Castellano. 2008. "Shape Memory Alloy Devices for the Structural Improvement of Masonry Heritage Structures." International Journal of Architectural Heritage 2(2):93-119.

[11] D'Ayala, D. F. and S. Paganoni. 2014. "Testing and Design Protocol of Dissipative Devices for Out-of-Plane Damage." Proceedings of the Institution of Civil Engineers: Structures and Buildings 167(1).

[12] Casciati, Fabio and Lucia Faravelli. 2009. "A Passive Control Device with SMA Components : From the Prototype to the Model." (June 2008):751-65.

[13] Paganoni, Sara. 2015. "Dissipative Anchor Devices for the Seismic Retrofit of Heritage Buildings." Ph.D. Thesis, University of Bath, Department of Architecture and Civil Engineering 1.

[14] Melatti, Victor and Dina D'Ayala. 2018. "Dissipative Device for the Seismic Protection of Masonry Structures." 16th European Conference on Earthquake Engineering 1-12.

[15] Lourenco, Paulo B., Gabriele Milani, Antonio Tralli, and Alberto Zucchini. 2007. "Analysis of Masonry Structures : Review of and Recent Trends in Homogenization Techniques 1." 1457:1443-57.

[16] Paganoni, S. and D. F. D’Ayala. 2012. "Numerical Simulation of Dissipative Anchor Devices in Historic Masonry." Proceedings of the Fifthteenth world Conference on earthqueake Engineering, Listbon, 2012 